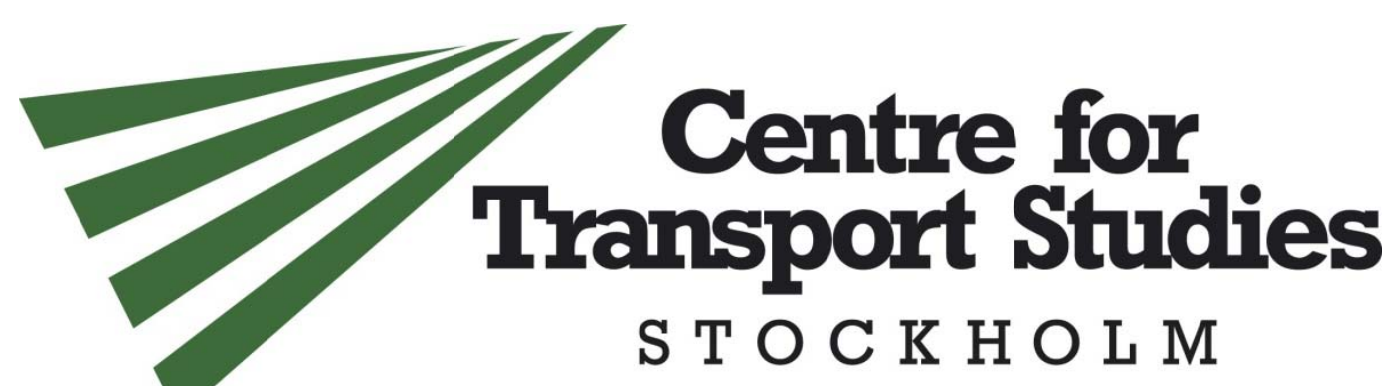

\title{
Pedestrians' behaviour in cross walks: The effects of fear of falling and age
}

\author{
Erel Avineri - University of the West of England, Bristol, UK \\ David Shinar - Ben Gurion University of the Negev, Israel \\ Yusak O. Susilo - Royal Institute of Technology (KTH)
}

CTS Working Paper 2011: 18

\begin{abstract}
Pedestrians are exposed to risks when crossing roads in urban areas. The crossing behaviour of pedestrians was studied as a factor contributing to their exposure to risks on the road and to their involvement in road accidents. This work explores two specific aspects of crossing behaviour: crossing speed and head pitches - the proportion of time pedestrians point their heads down (rather than towards the traffic) when crossing a road. The last one is used as an indicator of the (lack of) attention to cross-traffic. We also explored the possible effect of fear of falling (FOF) among pedestrians, as it might be associated with slow walking, less attention to cross traffic, and more attention to the pavement and their footsteps. This paper reports on a field study that combined an observatory technique with short survey. 203 pedestrians in two sites (signalised and unsignalised crosswalks) were video recorded while crossing the road. The FOF of pedestrians and other measures of pedestrian behaviour at crosswalks were revealed by means of questionnaire. Age and gender had the most significant effects on crossing speed, and FOF had a significant effect on the proportion of downward head pitches during crossing.
\end{abstract}

Keywords: Crossing behaviour, FOF (fear of falling), Age

JEL Codes: I00, O18, Z00

Centre for Transport Studies

SE-100 44 Stockholm

Sweden

www.cts.kth.se
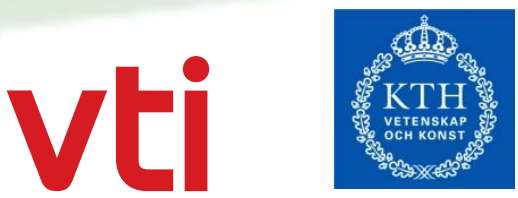



\title{
Pedestrians' behaviour in cross walks: The effects of fear of falling and age
}

\author{
Erel Avineri ${ }^{a}, *$, David Shinar ${ }^{b}$, Yusak O. Susilo $^{a}$ \\ ${ }^{a}$ Centre for Transport E' Society, University of the West of England, Frenchay Campus, Coldharbour Lane, Bristol BS16 1QY, UK \\ ${ }^{\mathrm{b}}$ Department of Industrial Engineering and Management, Ben Gurion University of the Negev, Ben-Gurion Avenue 1, P.O. Box 653, Beer Sheva 84105, Israel
}

\section{A R T I C L E I N F O}

\section{Article history:}

Received 14 June 2010

Received in revised form

26 November 2010

Accepted 27 November 2010

\section{Keywords:}

Crossing behaviour

FOF (fear of falling)

Age

\begin{abstract}
A B S T R A C T
Pedestrians are exposed to risks when crossing roads in urban areas. The crossing behaviour of pedestrians was studied as a factor contributing to their exposure to risks on the road and to their involvement in road accidents. This work explores two specific aspects of crossing behaviour: crossing speed and head pitches-the proportion of time pedestrians point their heads down (rather than towards the traffic) when crossing a road. The last one is used as an indicator of the (lack of) attention to cross-traffic. We also explored the possible effect of fear of falling (FOF) among pedestrians, as it might be associated with slow walking, less attention to cross traffic, and more attention to the pavement and their footsteps. This paper reports on a field study that combined an observatory technique with short survey. 203 pedestrians in two sites (signalised and unsignalised crosswalks) were video recorded while crossing the road. The FOF of pedestrians and other measures of pedestrian behaviour at crosswalks were revealed by means of questionnaire. Age and gender had the most significant effects on crossing speed, and FOF had a significant effect on the proportion of downward head pitches during crossing.
\end{abstract}

(C) 2010 Elsevier Ltd. All rights reserved.

\section{Introduction}

Understanding and analysing the risks pedestrians are exposed to when crossing a road has been the subject of many studies addressing aspects relating to the traffic, road design, traffic signals and road users' behaviours. There is extensive research on pedestrian behaviour and the evaluation of safety measures for pedestrians at urban areas. Two specific aspects of crossing behaviour that are studied in this work are pedestrians' crossing speed and head pitches-the proportion of time pedestrians have their heads down. Both measures are interesting because they might be associated with pedestrians' age and the so-called 'fear of falling' (that is associated by itself with older age) that has not been studied in the context of crossing behaviour.

The increasing proportion of older people in the community in industrialized countries (in many of them this proportion has reached $10 \%$ and above it), and the increase in their level of mobility and physical activity, make the safety of older road users an increasingly critical issue.

Older people are seen as a vulnerable group of road users. A wide range of factors has been examined in this context. Older people are those individuals who are most likely to be physi-

\footnotetext{
* Corresponding author. Tel.: +44 117 3283197; fax: +44 1173283002.

E-mail address: Erel.Avineri@uwe.ac.uk (E. Avineri).
}

cal vulnerable (DfT, 2001; Musselwhite, 2006). They experience deterioration in sensory and cognitive skills (Dunbar et al., 2004; Kovalchik et al., 2004; Salthouse, 1996), and a progressive loss of feeling independent (Orimo et al., 2006). Some or all of these factors might have affect on the crossing behaviour of older pedestrians.

Observational techniques have been widely used to understand crossing behaviour and identify risky behaviours of different age groups (see, for example, Oxley et al., 1997). The effects of agerelated attitudinal factors and their contribution to road crossing behaviour have been also addressed in the literature, but most of the research has focused on the risk taking attitudes of younger adults (Holland and Hill, 2007; Parker et al., 1992). There have been very few studies on the attitudes of older pedestrians towards risky behaviour. For an older pedestrian not paying enough attention to crossing traffic might be described as a risky behaviour. But, in general, older pedestrians exhibit safer behaviour when crossing a street (see for example Harrell, 1991). The hypothesis of high level of risk-taking among older pedestrians contradicts the general observation that risk taking decreases with age; older road users tend to take fewer risks than younger people in many different road safety contexts, and in road crossing in particular (Holland and Hill, 2007).

One shortcoming of older people is their slower gait. Older people walk more slowly when crossing the road (Coffin and Morrall, 1995; Oxley et al., 1997). Thus, the time spent by the pedestrian at crossing a road (so-called "the time of exposure") 


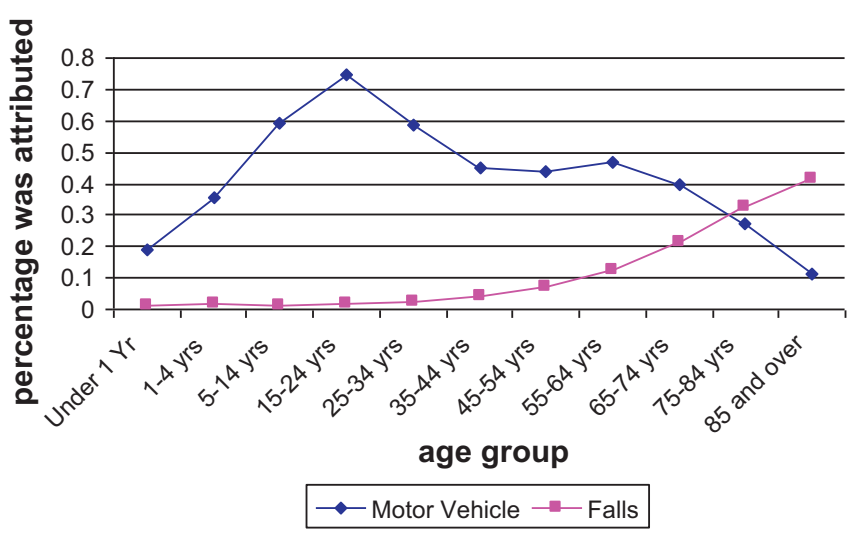

Fig. 1. Causes of accidental death in the US by age groups: falls vs. motor vehicles (based on CDC, 2002).

increases with age and increases risk exposure (Lassarre et al., 2007).

In addition to their vulnerability to traffic, people of all ages are exposed and vulnerable to falls. Cumming et al. (2000) define fear of falling (FOF) as a general concept that describes low confidence at avoiding falls, coupled with the greater fear of the consequences of falling. FOF is known to be multifactorial with, at a minimum, physical, psychological, and functional components. In older people it is associated with poorer health status and functional decline, increase in restriction of activity, psychological factors (such as depression and anxiety), and decreased quality of life (Legters, 2002). Diagnosing FOF has been the subject of at least 28 studies using a range of measurement methods (see review in Scheffer et al., 2008).

FOF has been largely associated with old age. Among people age 60 and older, falls are the leading cause of injury deaths (Stevens, 2005). More than a third of older people fall each year (Hausdorff et al., 1994), and about quarter of those who fall suffer moderate to severe injuries (Alexander et al., 1992). It is the third leading cause of death (after motor-vehicle accidents and 'unspecified non-transport accidents') in the U.S. (CDC, 2002). Fig. 1 shows the frequency of motor-vehicle accidents and falls as causes of death as a function of age: with the former peaking around age 20 and the latter increasing exponentially with age to the point where the pose a greater risk than motor-vehicle accidents at age $75+$.

FOF is a concern to $12-65 \%$ of older adults (see a review in Legters, 2002). Thus, it is possible that pedestrians who are more likely to be afraid of falling might walk slower than other pedestrians who are not. It could be also hypothesized that they are more likely to pay less attention to cross traffic, and pay more attention to the pavement and their footsteps when crossing a road-a behaviour that might compromise their safety.

Observational measures of FOF might provide additional explanation of crosswalk behaviour. For example, it could be associated with the speed of walking (which is expected to decrease with FOF). FOF could also be associated with a pedestrian's level of attention to cross traffic during crosswalking: the perceived risk of being involved, as a pedestrian, in a traffic accident might be traded-off with the psychological effect of FOF; pedestrians who are afraid of falling might pay less attention to the crossing traffic, and pay more attention to the pavement and to their footsteps.

It is difficult to capture pedestrians' eye movements in the field environment. Therefore, downward head pitches - specifically the proportion of time pedestrians have their heads pointed down (rather than towards the traffic) when crossing a road - is suggested as an indicator of the (lack of) a pedestrian's attention to crosstraffic. While the correlation between eye movements and head pitches have not been explored in the context of crossing behaviour, there is some evidence that they are related or even coordinated in a range of human and animal behaviours (Pratt, 1981; Guitton and Volle, 1987; Land, 1992; Gilchrist et al., 1997; Romoser and Fisher, 2009).

Large saccades are accompanied by rotations of the head (see Guitton, 1988 for a review). Moreover, according to Sparks (1991), the eyes and head of subjects move in the same direction only until the eyes reach their oculomotor limit or until the line of sight is directed at the target. Then, usually, the head continues to move and the eyes move in the opposite direction, compensating for the still ongoing head movement. It might be therefore argued that in such situations head movements could make be a good indicator of cognitive and visual attention.

Cross-traffic and other road hazards could be situated both within and beyond the oculomotor range human have (which is about $\pm 55^{\circ}$ ); therefore a certain frequency of head movements towards such targets during crossing would be expected.

Using a field study of crossing behaviour, we explored what measures of pedestrian behaviour explain their crossing speed and proportion of downward head pitches. We hypothesized that crossing speed is reduced, and proportion of downward head pitches during crossing is increased, with age and with FOF.

\section{Methods}

\subsection{Sites and participants}

The sites chosen for this study were a standard signalised crosswalk and a standard unsignalised crosswalk, both located in central Tel-Aviv, Israel.

Pedestrians' crossing speed and other aspects of crossing behaviour are affected by the walking environment, and by pedestrians' motivation and the purpose of the journey (Finnis and Walton, 2008). To ensure that the populations of pedestrians and drivers at both sites are similar as possible, the two crosswalks chosen for this study were located less than $50 \mathrm{~m}$ from each other.

There are no designed elements (such as humps) or enhanced features to assist disabled people at these crosswalks. The walking surfaces at both sites had essentially no gradient. Both crosswalks were clearly signed and marked (with zebra crossings). Maintenance conditions of the crosswalks were good (with no cracked pavements, potholes, etc. that might affect crossing behaviour). The speed limit in this urban area is $40 \mathrm{~km} / \mathrm{h}$.

The width of the signalised crosswalk is $10 \mathrm{~m}$. It crosses a twoway road. The length of the green light phase $(23 \mathrm{~s})$ should provide most pedestrians a reasonable amount of time to cross. At an average walking speed of $1.2 \mathrm{~m} / \mathrm{s}$ (commonly used to calculate timings at pedestrian crosswalks) it takes $5.8 \mathrm{~s}$ to cross the road. Most pedestrians, even those who walk at speeds less than $1 \mathrm{~m} / \mathrm{s}$, should have sufficient time to cross the road during the green phase. The unsignalised crosswalk is $6.6 \mathrm{~m}$ wide one-way street. By law, pedestrians crossing such road have priority over road traffic (although not all drivers in Tel-Aviv comply with this law). Both crosswalks are very close to a large supermarket and to a medical centre that attract people of a wide range of ages.

\subsection{Procedure and analysis}

This study used a mixed approach: an observational technique was applied to study the crossing behaviour of pedestrians at the two crosswalks, and a face-to face survey was held with pedestrians immediately after crossing.

Pedestrians crossing at both sites were randomly sampled. At both sites, the video camera was located at an unobtrusive fixed location on one side of the street. In order to make sure that 
Table 1

Age and gender frequencies of the pedestrians at the two sites.

\begin{tabular}{|c|c|c|c|c|c|c|}
\hline \multirow[t]{2}{*}{ Site } & \multirow[t]{2}{*}{ Number of pedestrians } & \multicolumn{2}{|c|}{ Gender } & \multicolumn{3}{|c|}{ Age group (years) } \\
\hline & & Male & Female & $18-35$ & $36-64$ & 65 and over \\
\hline Signalised crosswalk & 102 & 39 & 63 & 23 & 37 & 42 \\
\hline Unsignalised crosswalk & 101 & 29 & 72 & 26 & 42 & 33 \\
\hline Total & 203 & 68 & 135 & 49 & 79 & 75 \\
\hline
\end{tabular}

head pitches are well captured, only pedestrians crossing the street towards the location of the camera were included in the sample. In order to analyse the regular walking speed, when not crossing a road, pedestrians were video recorded walking on the sidewalk section near the crosswalk. All observations were made on a mid-week day between $10 \mathrm{am}$ and $2 \mathrm{pm}$. There were good weather conditions (bright sun, no rain) throughout the study.

After they crossed the road, face-to-face short interviews were held with the pedestrians. It included questions such as the pedestrian's age, marital status, and how often does the pedestrian cross this road. Pedestrians were asked about whether they were in a hurry when crossing the road, about whether they have been involved in road accidents, and whether they have vision problems. In addition, pedestrians were asked to describe their FOF on a 5-point Likert scale (possible responses were: I am not afraid of, usually I am not afraid of, I am a bit afraid of, I am afraid of, and I am much afraid of falling).

The data analysed in this study includes only those pedestrians who provided a full response to the questionnaire, and who provided their consent to be included in the study. The response level was about $60 \%$.

\section{Results}

Table 1 provides the number of pedestrians observed at each crossing as a function of age and gender.

\subsection{Crossing speed}

The effects of age, gender and the crosswalk type (signalised/unsignalised) on the walking speed of pedestrians at the two sites were examined with a linear regression model. Also examined were the pedestrians' responses to questions on being in a hurry, being involved in road accidents, having vision problems, and being afraid of falls. Because the proportion of pedestrians who reported any level of FOF was rather low (about $14 \%$ at all three levels of FOF, representing pedestrians who are "a bit afraid", "afraid" and "much afraid" of falling) this variable was dichotomized. Tests on multicollinearity did not show that any of the variables were significantly correlated with others.

The beta weights of the regression model are presented in Table 2. As expected age had a significant effect on the crossing speed: the crossing speed of younger adults (18-35) was significantly higher than the crossing speed of 36-64 years old

Table 2

The regression model for walking speed at the crosswalk.

\begin{tabular}{lcc}
\hline & $B$ & Sig. \\
\hline (Constant) & 1.450 & .000 \\
Female (dummy) & -0.74 & .056 \\
Age 18-35 & 0.116 & .013 \\
Age 65+ & -0.238 & .000 \\
In a hurry & 0.02 & .959 \\
Not involved in road accident & -0.070 & .304 \\
Afraid of falling & -0.075 & .198 \\
Unsignalised crosswalk & 0.02 & .979 \\
Has a vision problem & -0.013 & .714 \\
\hline
\end{tabular}

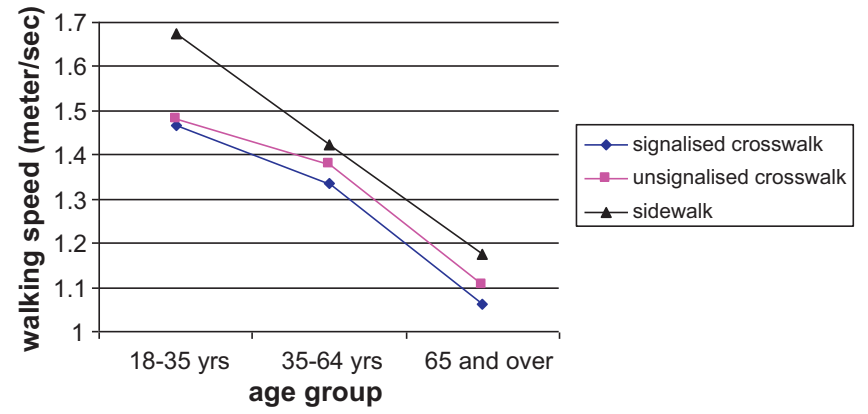

Fig. 2. Average walking speeds at the signalised crosswalk, unsignalised crosswalk, and the nearby sidewalk for different age groups.

pedestrians, who, in turn were faster than older pedestrians $(65+)$. The age effect was consistent in both crossings and in the 'normal' walking speed on the sidewalk, as illustrated in Fig. 2.

Other than age, the model also shows that females walk slower than males, though the relationship is marginally significant at $\alpha=10 \%$. None of the other variables has a significant effect on crossing speed. Specifically, we were unable to accept the hypothesis about crossing speed being associated with FOF.

Interestingly, Fig. 2 also indicates that pedestrians' speed is fastest on the sidewalk and slowest at the unsignalised crossing, but these differences are not significant.

\subsection{The proportion of downward head pitches during crossing}

The variables associated with the proportion of downward head pitches at the two sites were studied through a linear regression model, and the results are presented in Table 3. The variables included in the model were: crosswalk type (signalised/unsignalised), age, gender, and the responses of pedestrians to questions on their individual crossing-related experiences: being involved in road accidents, having vision problems, and being afraid of falls. However, the variables related to pedestrians' experience (made in response to the survey questions) turned out to be insignificant. This was not surprising; while there was not multicollinearity between variables, the inclusion of age made the influences of the experience-related variables weaker. In a model that included age the only variable that was marginally significant (at $\alpha=10 \%$ ) was FOF. Therefore, for the purpose of understanding the impact of the individual experience variables might have on the frequency of head pitches, the age factor has been excluded from the analysis presented in Tables 3 and 4.

Table 3

The regression model for downward head pitches during crosswalking.

\begin{tabular}{lrc}
\hline & \multicolumn{1}{l}{ B } & Sig. \\
\hline (Constant) & 0.117 & .121 \\
Female (dummy) & 0.040 & .295 \\
In a hurry & -0.030 & .403 \\
Not involved in road accident & 0.008 & .908 \\
Afraid of falling & 0.119 & .030 \\
Unsignalised crosswalk & -0.009 & .896 \\
Has a vision problem & 0.024 & .501 \\
\hline
\end{tabular}


Table 4

The regression model for downward head pitches at the midsection of the crosswalk.

\begin{tabular}{lrc}
\hline & \multicolumn{1}{l}{$B$} & Sig. \\
\hline (Constant) & -3.689 & .500 \\
Female (dummy) & 0.939 & .064 \\
In a hurry & -0.332 & .416 \\
Not involved in road accident & 1.259 & .110 \\
Afraid of falling & 1.113 & .026 \\
Unsignalised crosswalk & 1.207 & .125 \\
Has a vision problem & 0.215 & .593 \\
\hline
\end{tabular}

Table 3 shows that the only variable significantly associated with downward head pitch was FOF, with those admitting to FOF at some level spending more time looking at the pavement $(26.4 \%$ versus $14.0 \%$ ).

For a more detailed analysis of the phenomenon of looking at the pavement while crossing, we analysed the pedestrians' head pitches at three different segments of the crosswalks: at the start and at the end of the crosswalk, where pedestrians step down or up the sidewalk, and in the middle, i.e., the main section of the crosswalk. Table 4 shows the binary logit model result of whether the respondents' head faces down at the middle section of the cross ( 1 =head is down; $0=$ otherwise). Again, FOF was the only significant variable in the model (though the proportion of downward head pitches seem to be higher among female pedestrians at $\alpha=10 \%$ ).

None of the variables was found to be significant in the analysis of the proportion of downward head pitches at the first and third section of the crosswalk. This is probably because the beginning and end of the cross walk involve a perceptual and physical change that compels most people to attend to it (regardless of FOF).

\section{Study limitations}

There were several limitations to this study. It has been implied that a pedestrian's head pitches while crossing is indicative of his/her attention level to the cross-traffic, the pavements, his/her own footsteps, or other objects and movements in the walking environment. However head pitches might not be the best indicator of the direction in which her visual or cognitive attention is focused (though the two are generally correlated). Moreover, older pedestrians might suffer, more than people in other age groups, from physiological, sensory and cognitive limitations that might affect head movement and head pitches. For example, with advanced age the neck can become more stiff; coordination and speed of movements with the arms, hands and head is declining, and eye sight decreased (Isler et al., 1997; Doriot and Wang, 2006), among other factors, restrict the degree of freedom of head movements and affect the proportion of head pitches. Studying drivers' the frequency of side-to-side head turns while executing turns, Romoser and Fisher found that cognitive ability rather than physical factors as a significant predictor of head turns. It might be that such age-related factors, which were not tested in this study, and other controlled indicators of visual and cognitive attention (such as eye movements) would provide a significant contribution to the explanation of head pitches. This calls for a study of crossing behaviour in a more controlled environment (i.e., a simulation experiment rather than a naturalistic field study).

Also, it was sometimes difficult to estimate downward, forward, or towards traffic head pitches during crossing due to the fixed camera angle, crossing traffic and the direction pedestrians were walking in.

To obtain the pedestrians' cooperation the questionnaire was severely limited by length and included only 10 questions. Pedestrians' FOF has been assessed using a single question, on a rather general nature of FOF. Although directly derived from the definition of FOF, the responses to this question reveal nothing about the individuals' experience with falling, their risk perceptions, risk attitudes, and perceived behaviour control. Future research could explore how these measures might be linked to crossing behaviour, and which of them provide a better explanation and validation of FOF among pedestrians.

Finally, the study is limited by its scale. Lack of significance of some of the variables might be due to the relatively small number of observations within each cell in Table 1 . As it was not the main focus of this work, it was not attempted to draw specific conclusions on crosswalk design elements and their effect on crossing behaviour. Moreover, the two sites differ from each other in more than one dimension (the signalised intersection is a two-way road and the unsignalised intersection is a one-way street). Observing crossing behaviour in two sites provide only limited grounds for an understanding of crossing behaviour in a more general context, as a variety of factors in the crosswalk environment (such as surface level, lighting, volume of traffic, and safety measures) can be associated with pedestrians' crossing behaviour.

\section{Discussion and conclusions}

The safety of older pedestrians in the urban environment is a pressing issue for industrialized countries with an increasingly ageing demographic structure. The increase in conflicts between pedestrians and vehicles which results from the growth in motorised traffic in urban areas, calls for greater emphasis on improving the design of the walking environment for vulnerable pedestrians. By understanding the specific needs of vulnerable pedestrians, and their behaviour in the crossing of roads, crosswalks could be better planned and designed to improve their safety.

This study has shown that age differences and FOF have significant effects on pedestrian behaviour at crosswalks. Crossing speed is largely explained by age and gender, whereas the proportion of downward head pitches while crossing can be explained by gender and FOF (which by itself might be associated with old age).

Future research could also address the effect of age, gender, FOF and other relevant variables on other aspects of crossing behaviour, such as the choice of crossing location (i.e., where a pedestrian is more likely to cross) or their potential contribution to gapacceptance theory (Hamed, 2001; Manuszac et al., 2005; Lassarre et al., 2007).

Future research should incorporate findings and understanding of crosswalk behaviour into the design of interventions to treat FOF and gaze behaviour in both the transport and general contexts. Interventions to treat FOF in the planning and design of pedestrians' walking environment could be evaluated by their effects on walking speed, attention provided to cross traffic, head pitch, and - ultimately - number of accidents injuries and fatalities among older pedestrians while crossing the street.

\section{Acknowledgments}

The authors acknowledge the help provided by Noa Sobel and Liat Zoran in the collection of data. The authors would also like to thank the three anonymous reviewers for their insightful comments, which helped improve the content of this paper.

\section{References}

Alexander, B.H., Rivara, F.P., Wolf, M.E., 1992. The cost and frequency of hospitalization for fall-related injuries in older adults. American Journal of Public Health 82 (7), 1020-1023.

CDC, 2002. Deaths: Leading Causes for 2000. National Vital Statistics Report (NVSR) 50(16). Centers for Disease Control and Prevention, US. Available online: http://www.cdc.gov/nchs/products/nvsr.htm. 
Coffin, A., Morrall, J., 1995. Walking speeds of elderly pedestrians at crosswalks, Transportation Research Record 1487, 63-67.

Cumming, R.G., Salkeld, G., Thomas, M., Szonyi, G., 2000. Prospective study of the impact of fear of falling on activities of daily living, SF-36 scores, and nursing home admission. Journal of Gerontology A: Biological Sciences 55, M299-M305.

DfT, 2001. Older Drivers: A Literature Review. Department for Transport, London.

Doriot, N., Wang, X., 2006. Effects of age and gender on maximum voluntary range of motion of the upper body joints. Ergonomics 49 (3), 269-281.

Dunbar, G., Holland, C.A., Maylor, E.A., 2004. Older Pedestrians: A Critical Review of the Literature. DfT, London.

Finnis, K.K., Walton, D., 2008. Field observations to determine the influence of population size, location and individual factors on pedestrian walking speeds. Ergonomics 51 (6), 827-842.

Gilchrist, I.D., Brown, V., Findlay, J.M., 1997. Saccades without eye movements. Nature 390, 130-131.

Guitton, D., 1988. Eye-head coordination in gaze control. In: Peterson, B.W., Richmond, F.J. (Eds.), Control of Head Movement. Oxford Univ. Press, New York, pp. 196-207.

Guitton, D., Volle, M., 1987. Gaze control in humans: eye-head coordination during orienting movements to targets within and beyond the oculomotor range. Journal of Neurophysiology 58, 427-459.

Hamed, M.M., 2001. Analysis of pedestrians' behavior at pedestrian crossings. Safety Science 38 (1), 63-82.

Harrell, W., 1991. Precautionary street crossing by elderly pedestrians. International Journal of Aging and Human Development 32 (1), 65-80.

Hausdorff, J.M., Rios, D.A., Edelber, H.K., 1994. Gait variability and fall risk in community-living older adults: a 1-year prospective study. Archives of Physical Medicine and Rehabilitation 82 (8), 1050-1056.

Holland, C., Hill, R., 2007. The effect of age, gender and driver status on pedestrians' intentions to cross the road in risky situations. Accident Analysis \& Prevention 39 (2), 224-237.

Isler, R.B., Parsonson, B.S., Hansson, G.J., 1997. Age related effects of restricted head movements on the useful field of view of drivers. Accident Analysis \& Prevention 29 (6), 793-801.

Kovalchik, S., Camerer, C.F., Grether, D.M., Plott, C.R., Allman, J.M., 2004. Aging and decision making: a comparison between neurologically healthy elderly and young individuals. Journal of Economic Behavior and Organization 58 (2), 79-94.
Land, M.F., 1992. Predictable eye-head coordination during driving. Nature 359, 318-320.

Lassarre, S., Papadimitriou, E., Yannis, G., Golias, J., 2007. Measuring accident risk exposure for pedestrians in different micro-environments. Accident Analysis \& Prevention 39 (6), 1226-1238.

Legters, K., 2002. Fear of falling. Physical Therapy 82 (3), 264-272.

Manuszac, M., Manski, C., Das, S., 2005. Walk or wait? An empirical analysis of street crossing decisions. Journal of Applied Econometrics 20 (4), 529-548.

Musselwhite, C.B.A., 2006. Prolonging safe driving behaviour through technology: attitudes of older drivers. In: 26th International Congress of Applied Psychology , Athens, Greece, 16-21 July 2006.

Orimo, H., Ito, H., Suzuki, T., Araki, A., Hosoi, T., Sawabe, M., 2006. Reviewing the definition of "elderly". International Journal of Geriatrics Gerontology 6, 149158.

Oxley,J., Fildes, B., Ihsen, E., Charlton, J., Day, R., 1997. Differences in traffic judgments between young and old adult pedestrians. Accidents Analysis \& Prevention 29, 839-847.

Parker, D., Manstead, A.S.R., Stradling, S.G., Reason, J.T., 1992. Determinants of intention to commit driving violations. Accident Analysis \& Prevention 24 (2) 117-131.

Pratt, D.W., 1981. Saccadic eye movements are coordinated with head movements in walking chickens. The Journal of Experimental Biology 97, 217-223.

Romoser, M., Fisher, D.L., 2009. Effects of cognitive and physical decline on older drivers' side-to-side scanning for hazards while executing turns. In: Proceedings of the Fifth International Driving Symposium on Human Factors in Driver Assessment, Training and Vehicle Design.

Salthouse, T.A., 1996. The processing speed theory of adult age differences in cognition. Psychological Review 103, 403-428.

Scheffer, A.C., Schuurmans, M.J., Van Dijk, N., Van der hooft, T., De Rooij, S.E., 2008 Fear of falling: measurement strategy, prevalence, risk factors and consequences among older persons. Age and Ageing 37, 19-24.

Sparks, D.L., 1991. The neural control of orienting eye and head movements. In Humphrey, D.R., Freund, H.-J. (Eds.), Motor Control: Concepts and Issues. John Wiley \& Sons Ltd., Chichester, pp. 263-275.

Stevens, J.A., 2005. Falls among older adults-risk factors and prevention strategies. Journal of Safety Research 36, 409-411. 